\title{
Novel insights into parasite dynamics for leishmaniasis treatment
}

Neglected tropical diseases such as leishmaniasis have a huge impact on global health but are under-researched and under-funded. Treatment and diagnostic techniques rem expensive and difficult to implement in remote areas. Dr of the University of Sao Paulo, Brazil, has been conducting research into potential new therapeutic targets, focusing on exploiting the parasite's need to appropriate the nutrients

Neglected Tropical Diseases Ex
- eglected tropical diseases (NTDs) enlargement of the spleen and liver. are a disparate group of tropical Even with treatment, the fatality rate is infections caused by a variety or protozonn parastes. These, viruses, ar 'ntozoan parasites. These disease in 'neglected" due to a lack of public tend to proser most in impoverished areas where sanitation is poor and access to clean water and medicine is scarce. Despite their enigmatic nature, approximately one sixth of the global population (around one billion people) suffer with at least one NTD, which has a significant negative impact on quality of life. Dr Maria Fernanda Laranjeira-Silva and colleagues from the University of São Paulo, Brazil, have been investigating one particular NTD - leishmaniasis - in order to better understand its mechanisms and defin better therapeutic targets.

\section{LEISHMANIASIS}

\section{EPIDEMIOLOGY AND CAUSES}

(a paraitic, ingle celled orgnism) transmitted between mammals by the female phlebotomine sandfly and more than one million new cases emerge each year. Many species of sandfly (at least 90 transmit the disease. There are also 20 different species of parasite that cause the infection and at least 70 animal reservo that host them.

There are three main presentations of leishmaniasis: visceral leishmaniasis, cutaneous leishmaniasis and mucocutaneous leishmaniasis. Visceral leishmaniasis (VL), also known as kala-aza or black fever, is the most serious. VL is caused by L. donovani, L. infantum and L. chagasi. Symptoms include fever, skin estimated at 10-20\%

Cutaneous leishmaniasis (CL) is endemic in more than 90 countries. Symptoms that form at the site of inoculation by the sandfly and can van in severity Lesions caused by certain species of Leishmania such as $L$ infantum and $L$ tropica may spontaneously heal, post-infection. However, recovered patients who have been left with disfiguring scars often face social stigma and subsequent psychological suffering.

\section{Mucocutaneous leishmaniasis (MCL)} is an advanced stage of $C L$ and is severe, disabling and potentially lifethreatening. $90 \%$ of MCL occurs in Brazil, Bolivia, and Peru and is associated mainly with infection by L. braziliensis mose, and throt, loding to mutilation and/or obstruction of the nose, larynx and pharynx. MCL does not heal spontaneously and is often associated with bacterial co-infection like tuberculosis and pneumonia.

$V L$ and $C L$ infections can be initially asymptomatic which significantly increases the potential for transmission through factors such as migration of carriers and asymptomatic patients

LEISHMANIA

HOST-PARASITE INTERACTION As the parasite transfers from the midgut of the sandfly to the host macrophage of of white blood cell that 'eats' foreign species. It causes destruction of the

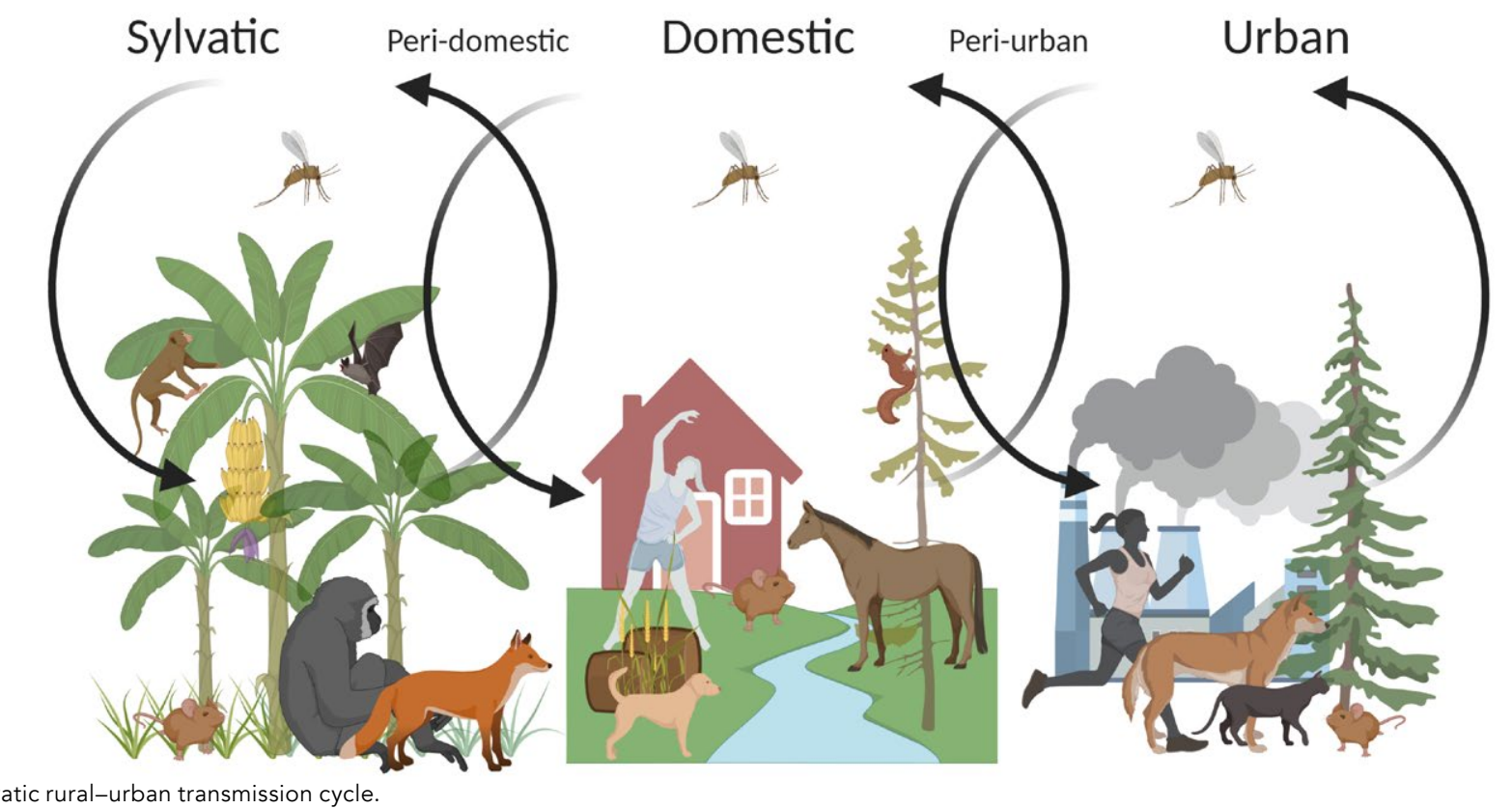

pathogens), it needs to obtain certain pathogh it is

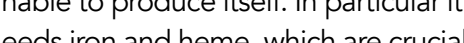
the growth and virulence of the paraste.

Further research focused on the impac of the primordial enzyme arginase (a ubiquitous enzyme) on the virulence The paraste must a cafully regulate of

virulence of leishmaniasis than previously hought. For example, arginase can alter everal host cell-signalling molecules, PPG is present on the surface of the parasite and facilitates the binding macrophage, as high Despite this promising new macrophage receptors, (damaging) effect due to research, there is currently no potential. Consequently $\begin{array}{ll}\text { their ability to generate } & \text { vaccine available for leishmaniasis. } \\ \text { Dr Laranjeira-Silva also } \\ \text { suggests the polyamines }\end{array}$

NOVEL LEISHMANIA

VIRULENCE STUDIES Dr Laranjeira-Silva and her colleagues have identified a novel iron-responsive L is the first proten to demonstrate is The researchers found that LIR 1 is critical to the virulence and norma growth of $L$. amazonensis. Results demonstrated that expression of $L I R$ in the parasite is regulated by iron levels - LIR1 expression increased when the parasite was exposed to excess iron levels and decreased with lower iron levels. This protects the parasite from accumulating toxic iron levels, but also ensures there is enough iron to promote virulence and healthy grow Dr Laranjeira-Silva proposes that the LIRI protein, and possibly other proteins of the iron-metabolism pathway, would the excellent therapeutic targets in the development of nw leishmanis freatments because of thir cirical

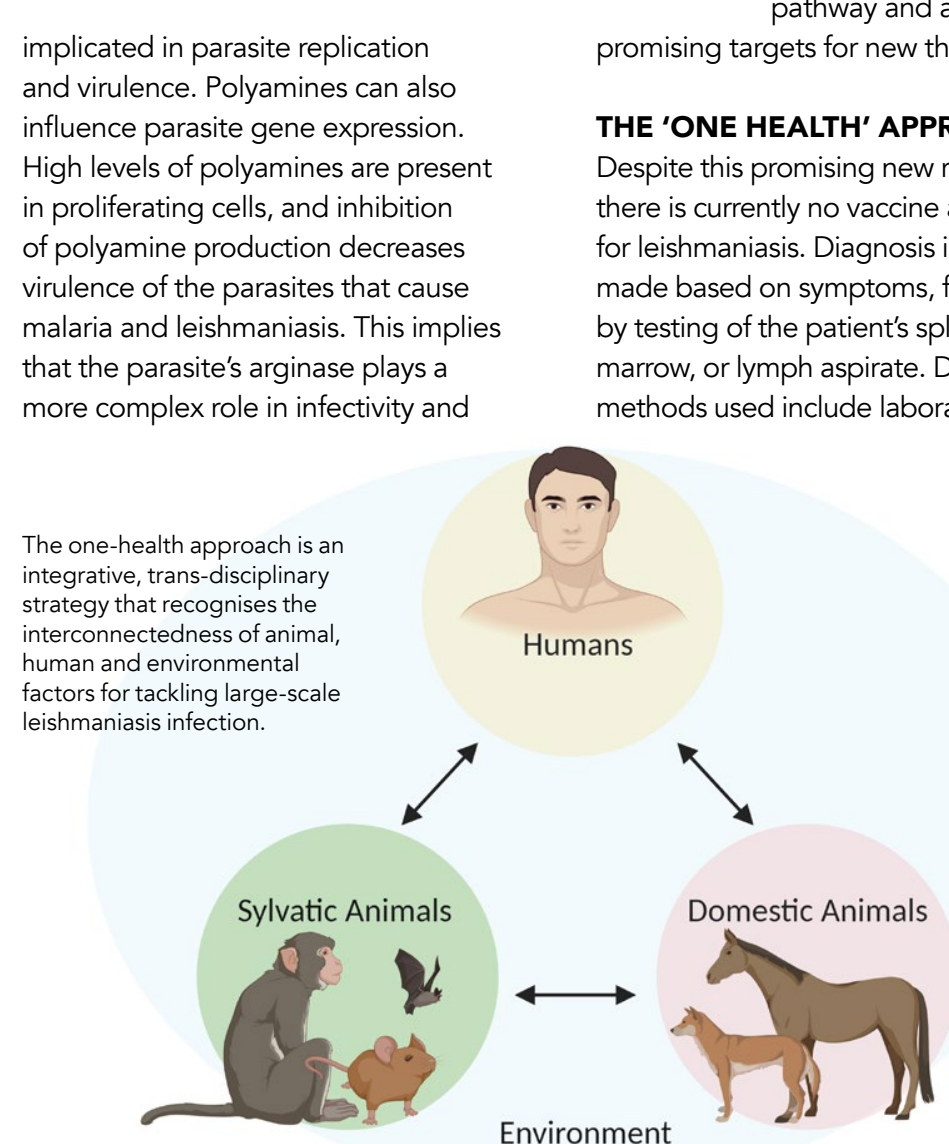




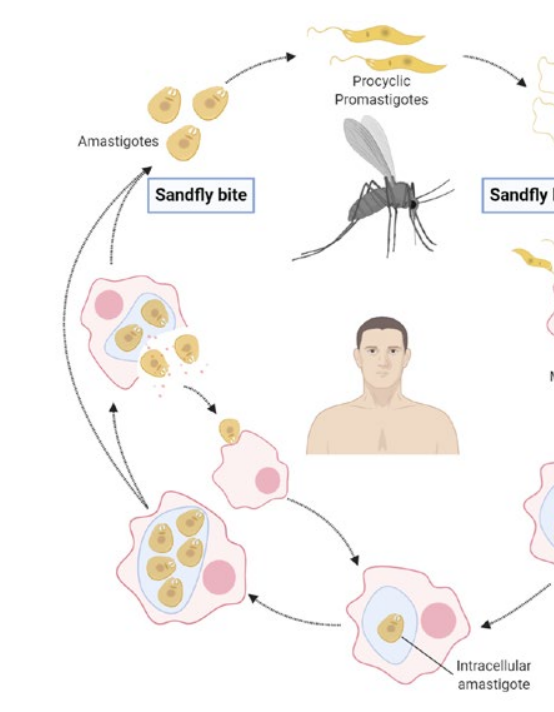

such as polymerase chain reaction (PCR). Early treatment significantly improves a patient's prognosis, but treatment remains expensive and implementing endemic countries and remote areas. The identification of the infecting species of Leishmania is also crucial.

To address these challenges, $\mathrm{Dr}$ Laranjeira-Silva proposes the adoption of the 'One Health' approach: an integrative, trans-disciplinary strategy that recognise the interconnectedness of animal, human and environmental factors for tackling large-scale leishmaniasis infection.

\section{SOCIO-ECONOMIC FACTORS} Leishmaniasis is common on almost every continent on the planet, however, the risk of contracting it is significantly higher for mpoverished communities who are unable to afford treatment or even bed nets. The results of a recentstudy in Nepal domonstrated that natural flooring materials increase the risk of contraction eightfold, while natural building materials tripled the likelihood of contracting leishmaniasis. Impoverished families are often malnourished, with weakened immune systems, further increasing their vulnerability

\section{POLITICAL AND}

\section{MIGRATORY INFLUENCES}

Several studies have demonstrated that there is a positive correlation between $\checkmark$ epidemics and political unrest, wi high infection rates occurring in war zones and densely populated areas such as refugee camps. Mass migration environmental change in Costa Rica was shown to increase the prevalence Furthermore the highest retes of zoon. leishmaniasis (passing of the disease from animals to humans) have been shown in communities that live close to an ecosystem that naturally harbours the disease, such as rainforests. For instance, Manaus, the largest urban centre in the Amazon rainforest, is home to 1.7 million people and accounts for more than $40 \%$ of leishmaniasis infections in Brazi.

\section{THE IMPORTANCE OF AN}

INTEGRATED APPROACH

The One Health approach is not a new idea; i thas gained popularity over recent years, however, due to its effectiveness in controlling various health issues such as food safety and ant microbial resistance, as well as the control ziconotic diseases The cornerstones on which the One Health approach is built include effective communication, co-ordination and collaboration among human-healthcar professionals, public-health officials, animal-health specialists and agricultural workers, as well as those who work in where the infection is less common.

The One Health approach recognises the interconnectedness of animal, human and environmental factors.

ENVIRONMENTAL RISK FACTORS Climate change and man-made alterations of the environment such as deforestaton also have a significant climate can affect the movement vectors and resenvir animals. Population increases in endemic regions requires mass deforestation to make way for housing. Deforestation-related

environmental fields, such as wildlife experts and ecologists.

This integrated approach, combined with the novel research that Dr Laranjeira-Silva and her team have produced, paves the way for important and sorely needed new avenues of treatment for this rampant and disease, giving new hope to millions of sufferers across the world.
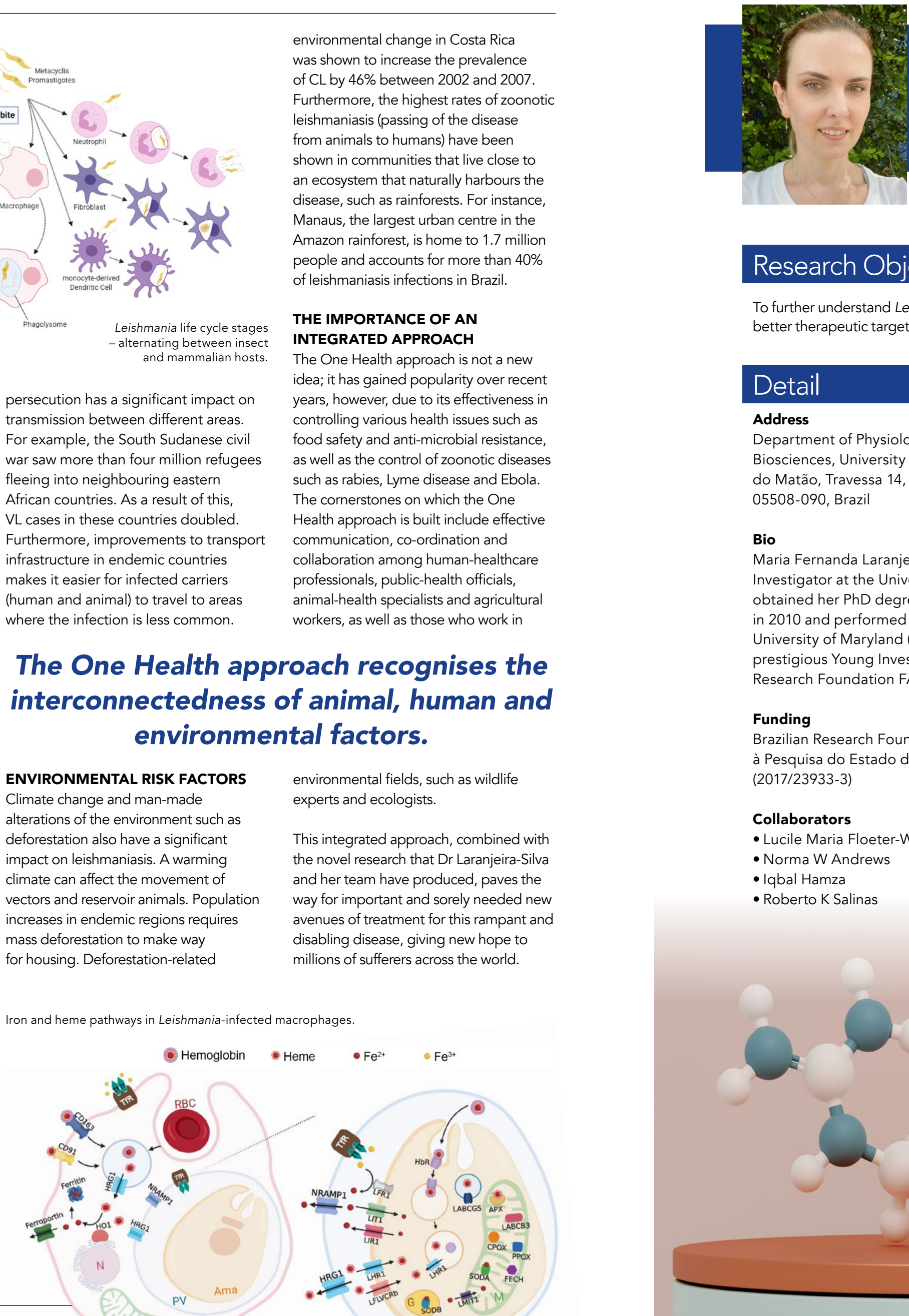

Behind the Research

\section{Dr Maria Fernanda Laranjeira da Silva}

E: mfernandals@usp.br E: mfernandals@gmail.com T: +551130917503 W: felab.science

\section{Research Objectives}

To further understand Leishmania pathogenesis to define better therapeutic targets for leishmaniasis treatment.

\section{Detail}

\section{Address}

Department of Physiology, Institute of do Matão, Travessa 14, 101, São Paulo SP, 05508-090, Brazil

Bio

Maria Fernanda Laranjeira-Silva is an Early-Stage Investigator at the University of Sao Paulo, Brazil She obtained her PhD degree from the same institution in 2010 and performed postdoctoral studies at the University of Maryland (USA), before receiving the prestigious Young Investigator Award from the Brazilian Research Foundation FAPESP in 2018.

Funding

Brazllan Research Foundation 'Fundação de Amparo à Pesquisa do Estado de São Paulo' (FAPESP) (2017/23933-3)

\section{Collaborators}

- Lucile Maria Floeter-Winter

- Norma W Andrews

- Roberto K Salinas

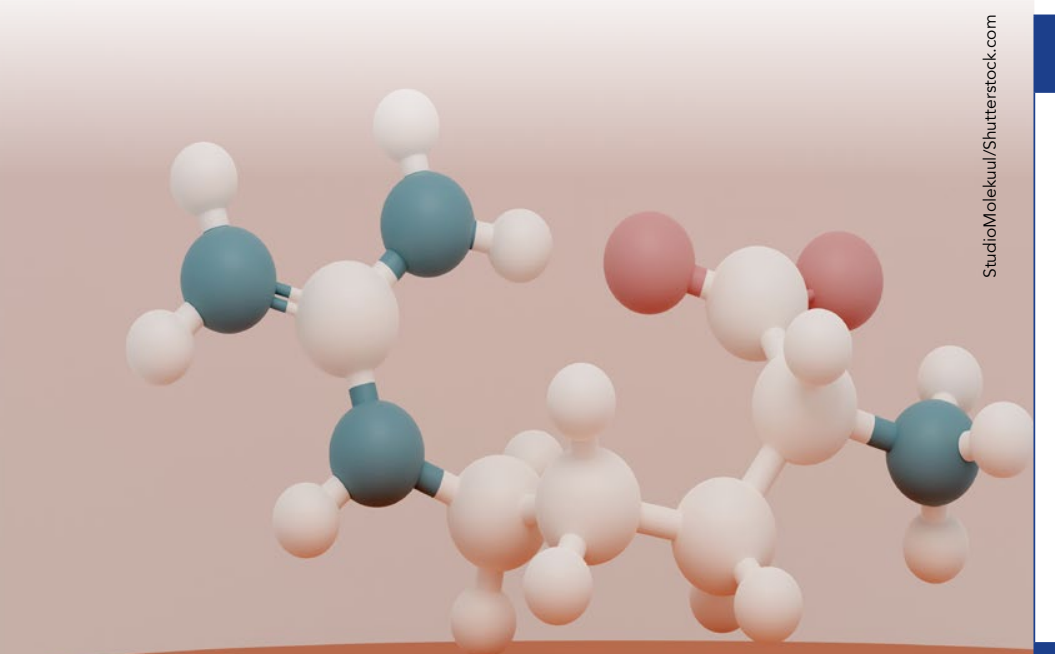

References

World Health Organisation (2021) Leishmaniasis Key Facts. [online] WHO. www.who.int/news-room/fact-sheets/detail/ leishmaniasis laccessed. 03/08/21

Hong, A, Zampieri, R, Shaw, J, Floeter-Winter, L, Laranjeira-

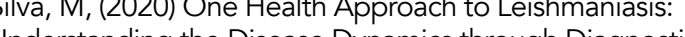
Understanding the Disease Dynamics through Diagnostic
Tools. Pathogens. 9 (809) 1-24. doiorg/10 $3390 /$ pathogens9100809

Aoki, J, Laranjeira-Sivaa, M, Muxel, S, Floeter-Winter, L, Aoki, J, Laranjeira-Silva, M, Muxel, S, Floeter-Winter, $L$,
(2019) The impact of arginase activity on virulence factors of Leishmania amazonensis. Current Opinion in Microbiology, Laranjeira-Silva, M, Iqbal, H, Pérez-Victoria, JM, (2019) Iron Trends in Parasitology 36(3). 279-289 doi.org 101016/ Trends in Parasitolo
pt.2019.12.010

Sarkar, A, Andrews, N, Laranjeira-Silva, M, (2019) Intracellular iron availability modulates the requirement for Leishmania Iron Regulator 1 (LIR1) during macrophage infections. Internationa Journal for Parasitol

Laranjeira-Silva, M, Wang, W, Samuel, T, et al, (2018) A MFS. Pathog, 14(6). doi.org/10.1371/journal.ppat.1007140 Centre for Disease Control and Prevention (2018). One Health Basics. [onlinel CDC. www.cdc.gov/onehealth/basics/index. html laccessed. 11/08/2021] National Institute of Allergy and Infectious Disease (2009) neglected Tropical Diseases Quick Facts. Lonline] NiH. wuw. niaid.nih.gov/research/neglected roptcal-diseases-quick-facts

\section{Personal Response}

What next steps are necessary to take you

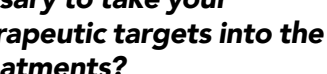

II Ongoing studies have been designed to further our which Leishmania acquires essential nutrients from the host for disease progression. Using this understanding we aim to establish these pathways as strong candidates for the design of molecules that specifically target parasite 3D structure determination of these parasite proteins will 3 sabrlecture determination of these parasite proteins will
alternative cheming of liagnds, which will be evaluated as
ales against leishmaniasis. 